\title{
Radiographic study of proximal ulna dorsal angulation in south Indian population
}

\author{
Reddy J.A.V. ${ }^{1}$, Garikapati S. ${ }^{2}$ \\ ${ }^{1}$ Dr. J. Ashok Vardhan Reddy, Associate Professor, Department of Orthopedics, Maheshwara Medical College \& \\ Hospital, Chitkul, Sangareddy District, Telangana, ${ }^{2}$ Dr. Sridhar Garikapati, Assistant Professor, Department of \\ Orthopedics, Shadan Institute of Medical Sciences \& Research, Centre, Peeranchetuvu, Himayathnagar Road, \\ Rangareddy District, Hyderabad, Telangana, India.
}

Corresponding Author: Dr. Sridhar Garikapati, Assistant Professor, Department of Orthopedics, Shadan Institute of Medical Sciences \& Research Centre, Peeranchetuvu, Himayathnagar Road, Rangareddy District, Hyderabad, Telangana.

\begin{abstract}
Background: The normal structure of proximal ulna is unique among long bones as described in the literature. Proximal dorsal angulation of ulna (PUDA) was not detailed in standard anatomical textbooks. The identification of PUDA is an important anatomic landmark for surgeons treating proximal ulna fractures, nonunions, malunions or osteotomies of proximal forearm in results of fractures. The study was conducted to identify the PUDA and to measure the distance from tip-to-apex of PUDA in bilateral elbow radiographs. Materials \& Methods: The present study was conducted on 56 bilateral elbow radiographs (26 Male, 30 Female), which are obtained from department of radiology. The radiographs were analyzed and measurements were recorded on each radiograph to identify the PUDA, later olecranon tip-to-apex distance of the PUDA was also measured. Results: No significant differences were observed in mean age of male and female patients. The measurements were recorded and entered in standard excel data sheet for analyzing PUDA in various patients. It was identified that $84 \%$ of the radiographs presents PUDA. Conclusion: Determination of the PUDA may be helpful in anatomic plating of the ulna for fractures, nonunions or malunions. Side to side correlation of PUDA with gender difference is reliable for recommendation to medical manufacturers for modelling ulna plates and components of artificial elbow joints.
\end{abstract}

Key words: Bilateral, Radiographs, Dorsal angulation, Ulna, Measurements

\section{Introduction}

The proximal ulna fractures results of severe injuries of proximal forearm often complicated because of the non union of fracture site. The use of anatomically preshaped ulna plates is observed in most of the proximal ulna fracture cases in recent years [1]. The medical manufactures make these plates purported to fit efficiently with structural anatomy of the proximal ulna [2]. But, several orthopedic surgeons reported based on their experience in surgical correction of proximal ulna fractures, these anatomically preshaped ulna plates do not fit perfectly for most of the patients.

The normal architecture of adult ulna is unique among long bones, which are described in the standard anatomical textbooks $[3,4]$. The dorsal angulation of proximal ulna has not been described in detail. The

Manuscript Received: $10^{\text {th }}$ June 2018

Reviewed: $20^{\text {th }}$ June 2018

Author Corrected: $28^{\text {th }}$ June 2018

Accepted for Publication: $3^{\text {rd }}$ July 2018 olecranon and the coronoid compose the greater sigmoid notch, which articulates with the trochlea. The lesser sigmoid notch, on the lateral aspect of the proximal ulna, articulates with the radial head to form the proximal radioulnarjoint. The articular surface of the greater sigmoid notch is covered with hyaline cartilage, except for a transverse "bare area" that divides the olecranon from the coronoid process. The recognition of Proximal Ulna Dorsal Agulation (PUDA) is crucial anatomic landmark for surgeons treating proximal ulna fractures while using the newer straight precountoured ulna plates.

These anatomically preshaped ulna plates also useful in non-unions, malunions or ostetomies of proximal forearm in results of fractures. Even though, some studies have already presented the data about the important landmarks of the PUDA, most of the authors were done in cadavers, unilateral elbow radiographs [5]. 


\section{Original Research Article}

Therefore, their reproducibility and applicability in the real surgical set up are debatable. Some of the newer studies were analyzed PUDA by using CT scan, which is not feasible for all the cases in real time surgery for measurements of PUDA.

On the other hand, if the surgeons use the nonanatomically precountered (straight) ulna plates to correct the proximal ulna fractures which will results alteration of force transmission and deform the structural integrity of the proximal ulna [6]. The structural alteration of proximal ulna may lead to dislocation of the radial head postoperatively.Therefore, it is essential to know the curvature in the posterior border of the proximal ulna in order to prepare or manufacture or select ideal anatomically preshaped ulna plates $[7,8]$.

The present radiographic study was undertaken to characterize the PUDA in patients with bilateral elbow radiographs and distribution based on gender and age in South Indians of Telangana state population. The details of the current study on proximal ulna anatomy withdetermination of PUDA may be useful for surgicalintervention involving plating of the proximal ulna, especially for selection precontoured proximal ulna plates.

\section{Materials \&Methods}

Place of the study: The present observational study was conducted in department of Orthopedics, MNR Medical College. The study was scrutinized and approved bythe Institutional Ethical committee.
Type of study: Observational study

Sampling method: Random method

Sample collection: Digital radiographs of lateral viewbilateral elbow joints were collected from the department of radiology; total 56 radiographs (26 Male, 30 Female) were obtained.

Inclusion Criteria: All radiographs were belonging to the patients aged between 19 to 68 years, and the mean age was 43 years.

Exclusion criteria: The quality of the radiographs were analyzed and identified by using the 3 concentric arcs of the trochlear sulcus, capitutlum and the medial trochlea.

Statistical Method: The measurements were recorded and entered in excel data sheet for analyzing PUDA in various patients.

Two orthopedic surgeons independently measured each radiograph to determine interobserver reliability. All radiographs were randomly arranged to reduce observer recall bias. The images were enlarged by using imaging software. The 'flat spot' was marked on the dorsal area of the proximal ulna. Dorsal angulation of ulna was determined by measuring the intersection angle tangent lines place on the 'flat spot' of the olecranon process and the dorsal ridge of the ulna shaft. Tip to apex distance of the PUDA was measured from the tip of the olecranon to the point of point of intersection of the PUDA tangent lines. The data was recorded patient age, side, diagnosis, PUDA, and tip- to apex distance.

\section{Results}

Table I shows correlation between left and right elbow joints with gender differences of PUDA. No significant differences were observed in mean age of male and female patients. The measurements were recorded and entered in excel data sheet for analyzing PUDA in various patients. It was identified that $84 \%$ of the radiographs presents PUDA. The average PUDA was $5.7^{\circ}$ on right elbow and $5.5^{\circ}$ on left elbow respectively. The range of PUDA in right elbow was 1.3 to 11.2 in males and 1.1 to 11.6 in females. The right elbow shows average tip-to-apex distance was $44 \mathrm{~mm}$ which is greater than left side $(41 \mathrm{~mm})$ in males. But, in females left elbow joint $(47 \mathrm{~mm})$ shows more average of tip-to-apex distance than right elbow joint $(42 \mathrm{~mm})$.

Table-I: Showing PUDA and tip-to-apex distance measurements of bilateral radiographs ofelbow joint.

\begin{tabular}{|c|c|c|c|c|c|c|c|}
\hline \multicolumn{2}{|c|}{ Measurements } & \multicolumn{3}{c|}{ Right } & \multicolumn{3}{c|}{ Left } \\
\cline { 3 - 9 } & Range & Average & SD & Range & Average & SD \\
\hline \multirow{2}{*}{ PUDA $^{\circ}$} & Male & $1.3-11.2$ & 5.7 & 2.3 & $0.3-11.2$ & 5.5 & 2.1 \\
\cline { 2 - 9 } & Female & $1.1-11.6$ & 5.6 & 2.6 & $0.4-11.4$ & 5.2 & 1.7 \\
\hline \multirow{2}{*}{ Tip-to-apex distance (mm) } & Male & $34.4-58.2$ & 44 & 5.3 & $31-62.7$ & 41 & 5.1 \\
\cline { 2 - 9 } & Female & $33-66.7$ & 42 & 6.2 & $32.1-61.4$ & 47 & 6.8 \\
\hline
\end{tabular}




\section{Original Research Article}

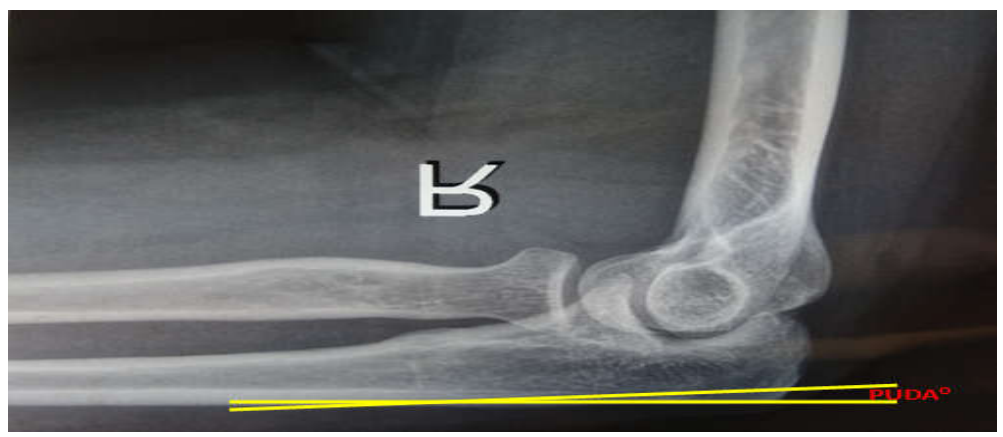

Figure-1: Lateral view radiograph of elbow joint showing PUDA between tangent lines placed on the dorsal aspect of the olecranon and the dorsal ridge of the ulnar shaft.

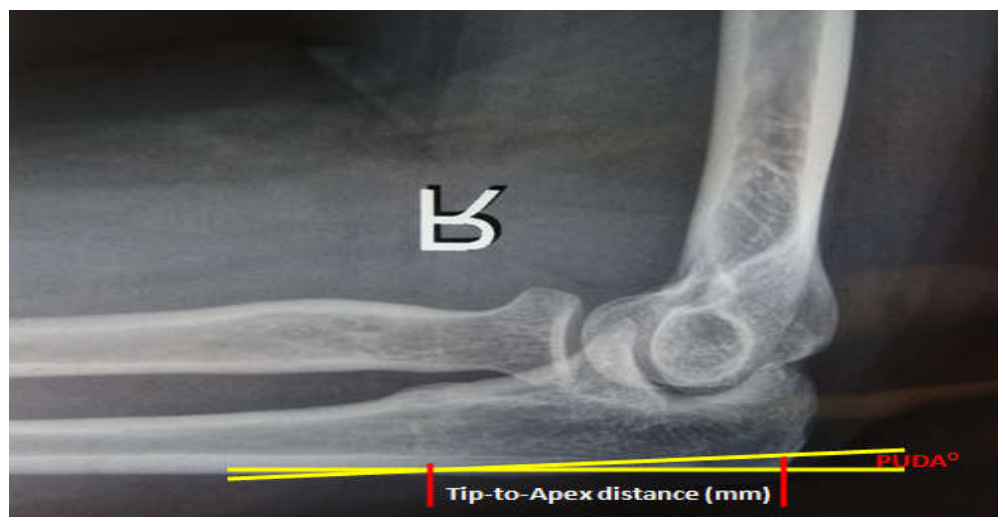

Figure-2: Lateral view radiograph of elbow showing location of the apex of the PUDA and tip-to-apex distance measurement.

\section{Discussion}

The unique bone structures in the proximal ulna present special difficulties in the reduction of an elbow joint, fracture fixation and arthroplasty. In the existing literature, the anatomy of the adult and adolescent ulna have been defined, however, there are few reports regarding the motion of the elbow and quality of life of the patient when these particular bone structures have been disrupted or deformed.

The computed images, cadavers are used for dorsal angulationof proximal ulna studies. Cadaveric studies have some limitations, as it does not give much detail about elbow and proximal ulnar anatomy. The reliability of measurements takenon cadaveric elbow is also questionable. Magnetic CT imagesare used for some studies have advantage over radiographicimages. Posterior border of the proximal ulna has been identified as an important anatomical landmark during surgical procedure for treating severe injuries of proximal forearm, especially for plate fixation in order to correct proximal ulna fractures. Fixing preshaped ulna plates has been widely accepted and followed by orthopedic surgeons in routine clinical practice. But, surgeons often struggle with fitting of the preshaped ulna plates because the shape of the plates differs from the dorsal angulation of ulna $[8,9]$. The straight architecture of ulnar plate will creates unbalanced forces in Monteggia fracture cases due to change in anatomical configurations of the proximal ulna and can result in postoperative dislocation of the radial head.

Therefore, the curvature in the posterior border of the proximal ulna should be acknowledged with PUDA and by measuring the tip-to-apex distance of PUDA. Even though, that structural anatomy of ulna itself is curved, it is crucial to recognize the PUDA for selection and manufacturing the plates for individual variation. To overcome these limitations, many manufactures have offered anatomically preshaped plates. But, these commercial plates are still unable to fit perfectly at the proximal ulna $[10,11]$. The PUDA data in south Indian population reported in the present study will enrich the database and improve the plate design. The data of current study suggest that the apex of dorsal angulation of ulnar in radiographs is located relatively more distal. When comparing the relationship between age and the 


\section{Original Research Article}

measured parameters, the tip-to-apex length of the ulna increased as age advanced, but age was not related to angles or percentage distances. The tip-to-apex distance of PUDA in left elbow were more in females as compared with males. Duggal $\mathrm{N}$ et al[12], reported mean PUDA of $5.7^{\circ}$ is present in $96 \%$ of patients with an average of $47 \mathrm{~mm}$ distal to the olecranon tip. The data was in agreement with present study. Significant variability in the side-to-side measurements of torsional alignment of the ulna with cross sectional magnetic resonance imaging was reported by Dumont et al[13].

The location and magnitude of the torsion was not reported in the present study. In a biomechanical study, reported that a $5^{\circ}$ malreduction at the PUDA already leads to radial head subluxation at the radiocapitellar joint. Thus, in complex fracture patterns, a contralateral elbow radiograph can be important to assess the patient's native PUDA because a straight locking plate may alter the normal anatomy and thus preclude successful radiocapitellar joint reduction.

Akpinar et al [14], described the anatomic factors of the proximal ulna to simplify intramedullary nail insertion for fracture fixation. The authors identified an optimum site for nail entry, intramedullary canal diameter, and canal length. The anatomic study with relevance to olecranon osteotomy and fracture fixation done on 39 paired elbows was studied by wang [15]. Ulnar length, the bare area of the sigmoid notch, lateral varus angulation, and ulnar cortical dimensions were reported in that study. The characteristics reported in the study were useful to assist the planning of olecranon osteotomies and fracture fixation.

Significant gender differences were found in tip-to-apex of the PUDA are in conformity with reports of Matzon et al [16], Mall et al [17], Wadia et al [18], described on radiographic measurement of normal elbows reported that gender differences for the PUDA were equivocal, with differences approaching statistical significance only on one side. Goldberg SH et al [19], studied on 54 cadaveric ulna and reported an average PUDA of 4.5, these findings are in comparison with the results of our study.

The current study shows high reliable PUDA measurements and the excellent side-to-side correlation with gender differences may permit us to make a recommendation to medical manufacturers for modeling ulna plates and other components of artificial elbow joints. The novel information in the study is sidetosidesymmetry, as the distance of the apex of the angulationfrom the olecranon tip.

\section{Conclusion}

The study summarizes that the PUDA radiographic study on bilateral elbow were important to recognize the anatomical curvature of proximal ulna. Both PUDA and tip-to-apex distance are important landmarks for orthopedic surgeons in order to applying plates and attempting to perform total elbow replacement surgery. The data reported in the current study is worthy to note down as most of the commercially available precontoured anatomical dorsal ulna plates are straight and it cannot fit during fixation of proximal ulna fractures. The observers recommended that radiographic image findingsalone are not sufficient while making treatment decisionsregarding the correction of rotational malunions, deformitiesdue to multiple hereditary exostosis.

Acknowledgement: Authors are thankful to department of radiology for providing radiographic data for the study.

Conflict of interest: None declared.

Funding: Nil, Permission from IRB: Yes

\section{References}

1. Brownhill JR, Mozzon JB, Ferreira LM, Johnson JA, King GJ. Morphologic analysis of the proximal ulna with specialinterest in elbow implant sizing and alignment. J Shoulder Elbow Surg 2009;18:27-32.

2. Hewins EA, Gofton WT, Dubberly J, MacDermid JC, Faber KJ, King GJ. Plate fixation of olecranon osteotomies. J Orthop Trauma 2007; 21(2):58-62.

3. Gray H. The ulna. Gray's Anatomy. 37th ed., Ch. 3, London, Churchill Livingstone Publishers, 1989. p. 413.

4. Kulkarni N. Elbow joint. In: Clinical Anatomy for Students;Problem Solving Approach. New Delhi: Jaypee BrothersPublishers; 2007. p. 63.

5. Warwick D. The elbow and forearm. In: Solomon L, Warwick D, Nayagam S, editors. Apley's System of Orthopaedics and Fractures. 8th ed. Hodder Arnold Publishers; 2001. p. 304.

6. Das S. A Manual of Clinical Surgery. 4th ed. S. Das's Publications; 1996. p. 139.

7. Laino DK, Petchprapa CN, Lee SK. Ulnar variance: correlation of plain radiographs, computed tomography, and magnetic resonance imaging with anatomic dissection. J Hand Surg Am. 2012;37:90-97. 


\section{Original Research Article}

8. Dhillon MS, Gopinathan NR, Kumar V. Misconceptions about the three point bony relationship of the elbow. Indian J Orthop2014;48:453-7.

9. Grechenig W, Clement H, Pichler W, Tesch NP, Windisch G. The influence oflateral and anterior angulation of the proximal ulna on the treatment of a Monteggia fracture: an anatomical cadaver study. J Bone Joint Surg Br.2007 Jun;89(6):836-8.

10. Windisch G, Clement H, Grechenig W, Tesch NP, Pichler W. Amorphometrical study of the medullary cavity of the ulna referred tointramedullary nailing. Surg Radiol Anat. 2007 Feb; 29(1):47-53.

11. Puchwein $\mathrm{P}$, Schildhauer TA, Schöffmann S, Heidari N, Windisch G, PichlerW. Three-dimensional morphometry of the proximal ulna: a comparison tocurrently used anatomically preshaped ulna plates. J Shoulder Elb Surg.2012;21:1018-23.

12. Duggal N, Dunning CE, Johnson JA, King GJ. The flat spotof the proximal ulna: a useful anatomic landmark in totalelbow arthroplasty. J Shoulder Elbow Surg 2004;13:206-7.

13. Dumont CE, Pfirrmann CW, Ziegler D, Nagy L. Assessment of radial and ulnar torsion profiles with cross-sectional magnetic resonance imaging. A study of volunteers. J Bone Joint Surg Am 2006; 88: 1582-8.
14. Akpinar F, Aydinlioglu A, Tosun N, Tuncay I. Morphologic evaluation of the ulna. Acta Orthop Scand 2003; 74:415-9.

15. Wang AA, Mara M, Hutchinson DT. The proximal ulna: An anatomic study with relevance to olecranon osteotomy and fracture fixation. J Shoulder Elbow Surg 2003;12:293-6.

16. Matzon JL, Widmer BJ, Draganich LF, Mass DP, Phillips CS. Anatomy of the coronoid process. J Hand Surg [Am] 2006; 31:1272-8.

17. Mall G, Hubig M, Buttner A, Kuznik J, Penning R, Graw M. Sex determination and estimation of stature from the long bones of the arm. Forensic Sci Int 2001;117:23-30.

18. Wadia F, Kamineni S, Dhotare S, Amis A. Radiographic measurements of normal elbows: clinical relevance to olecranon fractures.Clin Anat 2007; 20: 407-10.

19. Goldberg SH, Omid R, Nassr AN, Beck R, Cohen MS. Osseous anatomy of the distal humerus and proximal ulna: implications for total elbow arthroplasty. J Shoulder Elbow Surg 2007; 16 (3 suppl): S3946.

\section{How to cite this article?}

Reddy J.A.V, Garikapati S. Radiographic study of proximal ulna dorsal angulation in south Indian population. Surgical Update: Int J surg Orthopedics.2018;4(3):110-114.doi:10.17511/ijoso.2018.103.03. 